\title{
In-situ neutron PDF measurements of material in transformation: MXene and ferrite case studies
}

\author{
$\underline{\text { Peter C. Metz }}^{* a}$ and Katharine Page ${ }^{* b}$ \\ "Neutron Scattering Division, Oak Ridge National Laboratory, Oak Ridge, TN 37830, USA. \\ ametzpc@ornl.gov \\ bpagekl@ornl.gov
}

In-situ scattering experiments are a powerful tool, yielding detailed atomic structural information correlated to external stimuli. While $3^{\text {rd }}$ generation synchrotron $\mathrm{X}$-ray sources have enabled many novel in-situ capabilities, the relatively low fluence of neutron sources and the differing materials interaction mechanisms present unique hurtles to analogous neutron scattering experiments. The NOMAD team at the Spallation Neutron Source has recently developed a gas flow cell operable up to $600^{\circ} \mathrm{C}$ and are currently developing a TiZr hydrothermal pressure cell for the observation of nucleation, growth, and phase evolution under various hydrothermal conditions. These capabilities will be showcased by in-situ neutron diffraction and atomic pair distribution function (PDF) measurements of hydrothermal ripening of the ferrite $\mathrm{Bi}_{2} \mathrm{Fe}_{4} \mathrm{O}_{9}$ which exhibits sizedependent ferromagnetism at the nanoscale, and of in-situ nitridation in a two-dimensional MXene $\mathrm{Mo}_{2} \mathrm{C}$. The hydrothermal experiment will push the detection limit achievable on NOMAD, with an estimated $0.1-1 \%$ of the incident neutron flux resulting in coherent scattering from the nucleating phase. Particle size evolution will be tracked by neutron diffraction, while evidence of a ferromagnetic state will be inferred from the atomic PDF. Meanwhile, the gas flow-cell experiment will provide the first in-situ observation of MXene nitridation, which is one of the only synthesis routes towards two-dimensional nitride nanosheets. This work advances both in-situ NPDF methodology and the materials knowledge of these multifunctional materials under transformation. 